\title{
Endolymphatic Sac Tumor Associated with a Von Hippel-Lindau Disease Patient: An Immunohistochemical Study
}

Hidehisa Horiguchi, M.D., Ph.D., Toshiaki Sano, M.D., Ph.D. Hiroyuki Toi, M.D., Teruyoshi Kageji, M.D., Ph.D. Mitsuyoshi Hirokawa, M.D., Ph.D., Shinji Nagahiro, M.D., Ph.D. Departments of Pathology and Neurosurgery, University of Tokushima School of Medicine, Kuramoto, Tokushima, Japan

The authors report a case of endolymphatic sac tumor (ELST) associated with Von Hippel-Lindau disease (VHL). A 20-year-old female VHL patient received a resection of a cerebellar hemangioblastoma 3 years ago and she had a co-existing of left petrous tumor. The petrous tumor showed a remarkable progression in 3 years and was resected subtotally. Histologically, the resected petrous tumor showed a papillary structure containing cuboidal or columnar cells with fibrous stroma and numerous microvessels and destructed temporal bone, all of which are consistent with ELST. We studied the expression of various kinds of cytokeratins (CKs) immunohistochemically and found distinct expression of CKs (CAM 5.2, 34ßE-12, CK7, CK8 and CK19), but not for CK10/13 or CK20. Vascular endothelial growth factor and neuron specific enolase showed strong immunoreactivity in the tumor cells. CD34 also had weak expression. Ki-67 antigen (MIB-1) immunoreactivity was found in focal areas, and the labeling index in the highestdensity area was $48.9 \%$. These findings suggest that vascular endothelial growth factor overexpression is an important factor for angiogenesis in ELST, much like other VHL-associated tumors, and that ELST may have a more highly aggressive component than the low-grade malignancy noted in previous reports.

KEY WORDS: Endolymphatic sac tumor, Aggressive papillary tumor of temporal bone and endolym-

Copyright (C) 2001 by The United States and Canadian Academy of Pathology, Inc.

VOL. 14, NO. 7, P. 727, 2001 Printed in the U.S.A.

Date of acceptance: April 3, 2001.

Address reprint requests to: Hidehisa Horiguchi, M.D., Ph.D., Department of Pathology, University of Tokushima School of Medicine, 3-18-15 Kuramoto, Tokushima, 770-8503, Japan; e-mail: hide@basic.med.tokushima-u.ac.jp; fax: 88-633-9423. phatic sac, Von Hippel-Lindau disease, Ki-67, angiogenesis.

Mod Pathol 2001;14(7):727-732

Endolymphatic sac tumor (ELST) (1) is a rare neoplasm arising from the temporal petrous region. The origin of this tumor is thought to be the endolymphatic sac $(1,2)$. ELST histologically shows epithelial features, such as papillary architecture, glandular formation and a colloid-like structure. Immunohistochemical expression of cytokeratins (CKs) has been noted in ELST, and, thus, this tumor is believed to be an epithelial tumor (2-8). Because ELST is radiographically hypervascular, preoperative misdiagnoses of paraganglioma, glomus tumor and other temporal tumors occasionally have been given (1). ELST must be differentiated histologically from these tumors occurring in petrous bone $(2,3$, $6,9-14)$.

Von Hippel-Lindau disease (VHL) is a hereditary multi-tumor syndrome. Many hypervascular tumors, such as hemangioblastomas, renal cell carcinomas, and pheochromocytomas, are known to be manifestations of VHL. Recently the fact that ELST also has a significant association with VHL has been recognized $(2,6,15-17)$, and several VHL patients have presented with bilateral ELSTs $(6,7)$. This suggests that tumorigenesis of ELST is relevant to the genetic aberration of VHL.

Genetically the VHL gene has been identified as a tumor suppressor gene in cell lines of VHL patients and sporadic renal cell carcinomas (18). This gene regulates vascular endothelial growth factor (VEGF), and there is evidence that inactivation of VHL promotes VEGF overexpression and angiogenesis of renal cell carcinomas and hemangioblastomas $(19,20)$. However, VEGF expression in ELST has not yet been documented.

Biologically, ELST was previously considered a low-grade adenocarcinoma (1). It often shows invasion to surrounding temporal bone $(3,4,12)$ and 
there are reports of several patients who received incomplete resection and experienced a recurrence of the tumor $(7,21)$, although proliferative activity has been unknown in most ELSTs.

We encountered a case of ELST associated with VHL. In this study, we studied the tumor's proliferation and angiogenesis to assess its biologic behavior.

\section{CASE REPORT}

A 20-year-old woman received a resection of a hemangioblastoma of the cerebellar vermis (Fig. 1) 3 years ago and she was simultaneously pointed out to have a pancreatic cyst on ultrasonic examination. Her mother and elder brother also had history of resection of cerebellar hemangioblastomas. Therefore, she was clinically recognized to have
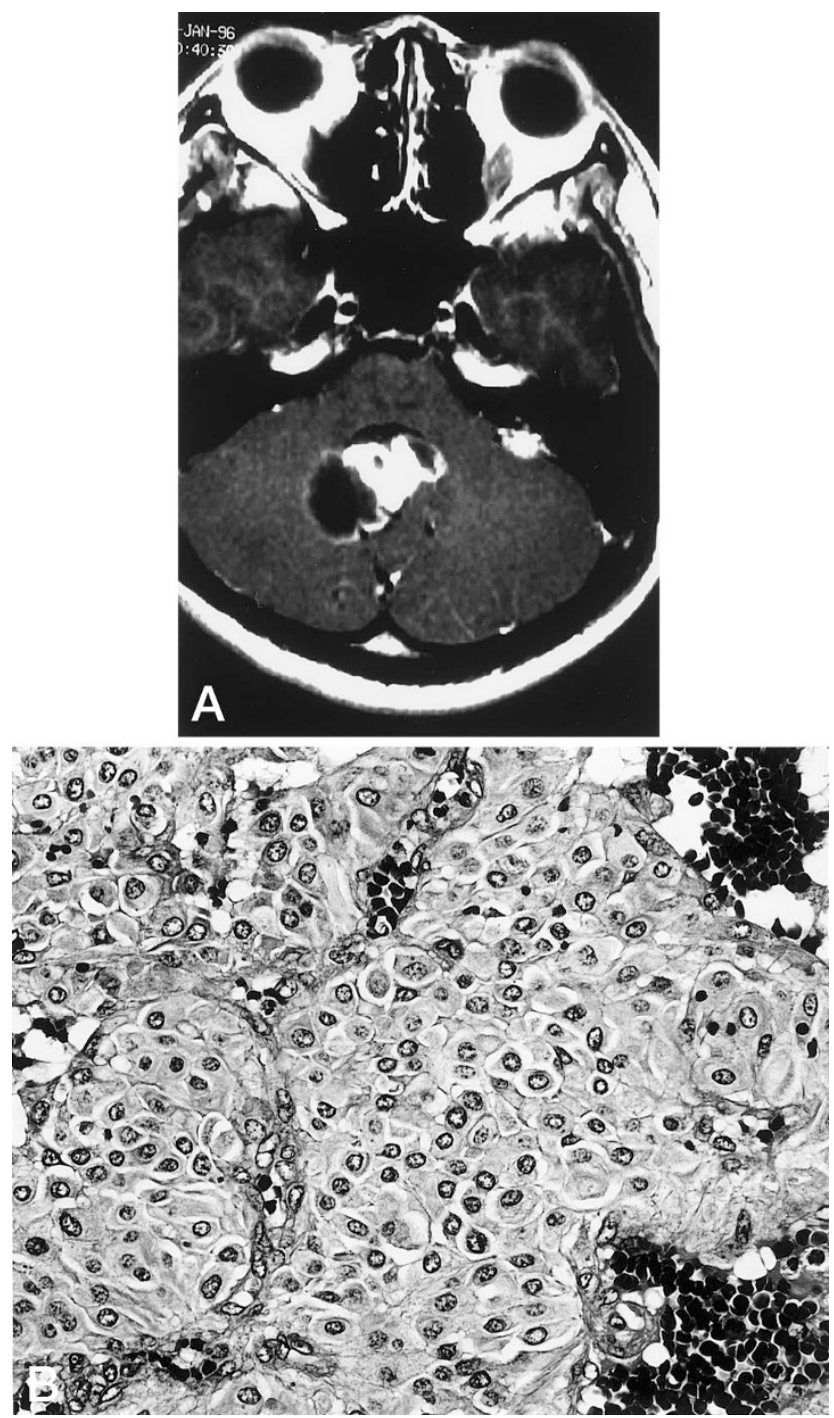

FIGURE 1. A, MRI showing a tumor with a cyst of the cerebellar vermis and a small tumor of the left petrous region. B, Histology of the tumor of the cerebellar vermis showing typical feature of hemangioblastoma.
VHL. At the time of initial surgery for cerebellar hemangioblastoma, a left temporal (medial petrous) tumor with approximate $1 \mathrm{~cm}$ in diameter was also noticed on magnetic resonance imaging (MRI, Fig. 1). Then, the petrous tumor had been carefully followed radiographically, and 3 years later after the surgery MRI revealed progression of the petrous tumor involving temporal bone (Fig. 2). Computed tomography (CT) scan showed destruction and honeycomb-like deformity of the left petrous bone (Fig. 2). The patient had lost her left hearing. After the embolization of the feeding arteries, surgery was performed for the petrous tumor. Macroscopically the petrous bone showed honeycomb-like deformity. A brown fibrous tumor existed in the petrous bone involving semicircular ducts, and invaded the jugular vein and subdural space in the cerebello-pontine angle. The tumor was removed subtotally. A radical resection of the petrous bone was not performed. After the surgery the patient had mild hoarseness. Postoperative MRI showed the residual tumor in the petrous region. Follow-up MRI taken 19 months after the surgery demonstrated regrowth of the tumor. No evidence of metastasis has been seen.

\section{MATERIALS AND METHODS}

Tissue was fixed in $10 \%$ formalin and embedded in paraffin. Sections $4 \mu \mathrm{m}$ thick were prepared for hematoxylin and eosin stain, Periodic-acid Schiff (periodic acid-Schiff) reaction, and immunohistochemistry. Antibodies for immunohistochemistry used were as follows: CKs (CAM 5.2, BectonDickinson, Mountain View, CA, diluted 1:1; CK7, DAKO, Glostrup, Denmark, 1:50; CK8, DAKO, 1:50; CK 10/30, DAKO, 1:50; CK19, DAKO, 1:50; CK20, DAKO, 1:50; 34ßE-12, Enzo, NY, 1:50), epithelial membrane antigen (EMA, DAKO, 1:100), carcinoembryonic antigen (CEA, DAKO, 1:50), CA19-9 (DAKO, 1:50), E-cadherin (Becton-Dickinson, 1:500), glial fibrillary acidic protein (GFAP, DAKO, 1:500), S-100 protein (DAKO, 1:500), chromogranin A (DAKO, 1:300), synaptophysin (DAKO, 1:100), neuron specific enolase (DAKO, 1:300), thyroglobulin (DAKO, 1: 2000), vimentin (DAKO, 1:20), CD34 (Becton Dickinson, 1:30), VEGF (Santa Cruz Biotechnology, Santa Cruz, CA, 1:100) and Ki-67 antigen (MIB-1, Immunotech, Marseilles, France, 1:50). The specimen for MIB-1 was pretreated by autoclave. Microwaves were used for activation of epitopes of CK7, CK10/13, CK19, CK20, EMA, CEA, E-cadherin, chromogranin A, synaptophysin, neuron specific enolase, vimentin, CD34 and VEGF. Immunoreaction was performed using the labeled streptavidin-biotin method, and diaminobenzidine (3,3'-diaminobenzidine) was used for visualization. 



FIGURE 2. A, MRI showing a growth of the left petrous tumor involving the temporal bone. B, CT scan showing destruction of the left temporal bone. C, Three dimensional CT scan showing honeycomb-like deformity of the left temporal bone.

For MIB-1, the rate (\%) of immunoreactive nuclei in 1000 cells in the highest-density area under highpower view was designated as the labeling index (LI).

\section{RESULTS}

\section{Pathologic Findings}

Microscopically, the tumor was composed of non-ciliated columnar or cuboidal cells lining one layer and mainly showed a papillary structure (Fig. 3). Glandular structures were also noted, and some of them contained colloid-like fluid. Tumor cells had eosinophilic and occasionally clear cytoplasm and a round to oval nucleus without atypia. There was a fine granular chromatin pattern. Neither nuclear inclusion nor mitosis was seen. No necrosis was noted. The stroma consisted of thick fibrous connective tissue and numerous microvessels. Hemorrhage and hemosiderin deposits were frequently seen. The tumor infiltrated into the petrous bone and destroyed it. Some tumor cells contained globules in periodic acid-Schiff reaction (Fig. 4). These morphologic features were consistent with ELST.

\section{Immunohistochemistry}

Immunohistochemically the tumor cells expressed strong reactivity for CKs: CAM5.2, CK7, CK8, CK19 (Fig. 5) and 34ßE12 but negative reactivity for CK10/13 and CK20. Immunoreactivity for EMA and vimentin was also demonstrated. Focal areas showed immunoreactivity for E-cadherin. No reactivity was recognized for CEA or CA 19-9. CD34 showed weak expression. Strong expression of VEGF (Fig. 6) was seen in the cytoplasm of the tumor cells. GFAP, S-100 protein, synaptophysin, chromogranin A and thyroglobulin had no immunoreactivity. Although most areas showed no immunoreactivity for Ki-67 antigen, focally a few positive areas were identified, and the LI in the highestdensity area was $48.9 \%$ (Fig. 7 ).

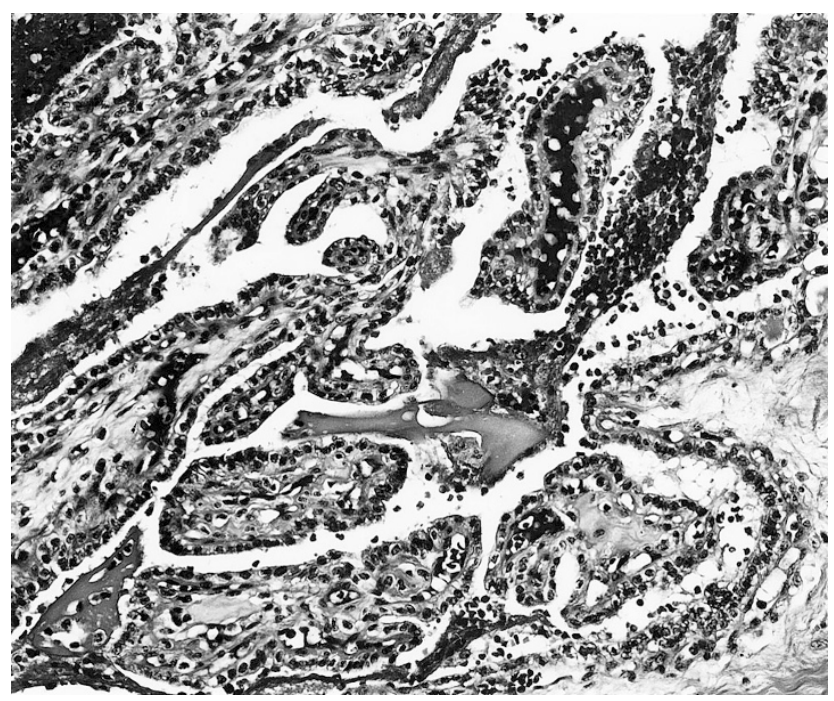

FIGURE 3. Endolymphatic sac tumor showing papillary structure composed of cuboidal cells. 


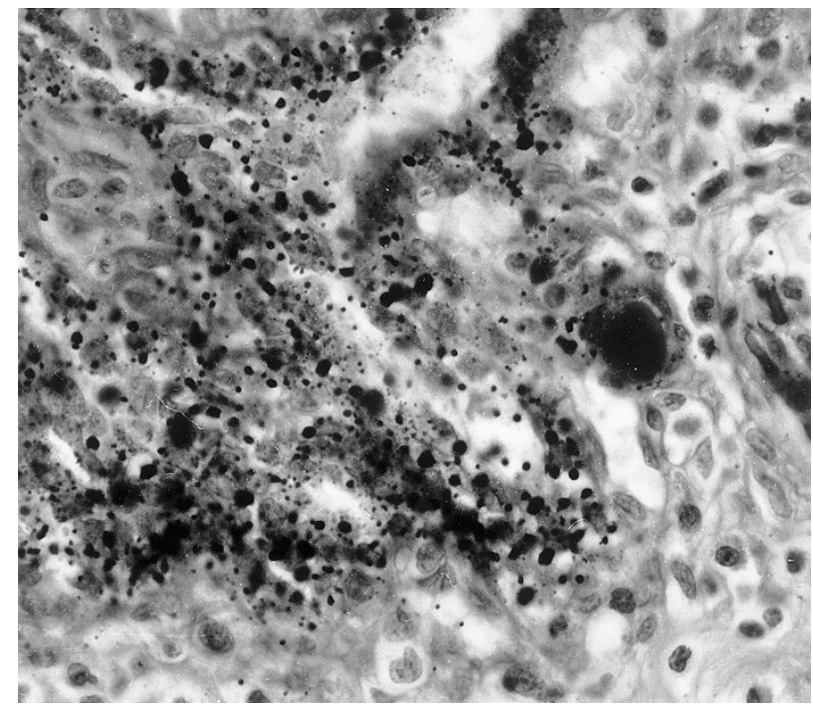

FIGURE 4. Endolymphatic sac tumor showing numerous periodic acid-Schiff reactive globules.

\section{DISCUSSION}

Heffner (1) postulated 20 cases of temporal bone tumor as low-grade adenocarcinoma of probable endolymphatic sac origin (so-called Heffner's tumor) with detailed clinicopathologic data. Recently Wenig and Heffner (2) added radiologic and intraoperative features and histogenesis for these tumors. They considered the origin of the tumors to be the endolymphatic sac, based on the histologic similarity between the tumor and a normal endolymphatic sac and clinical manifestations, hearing loss, tinnitus and vertigo. With these features they proposed ELST as a distinct new entity. Normal endolymphatic sac shows papillary epithelium composed of cuboidal or columnar cells and has neuroectodermal origin (22). ELST histologically corresponds to this feature and, therefore, has been supposed to express neuroectodermal markers (2, 12). Immunoreactivity for neuron specific enolase in the present case supports this hypothesis.

The nomenclature of aggressive papillary tumor of temporal bone and endolymphatic sac (APTTE) has been recently defined as tumors with papillary architecture and potential sites of origin elsewhere in the temporal bone (23). According to the definition, APTTE does not seem to be a distinct entity because it includes such as adenoma or adenocarcinoma of the middle ear, aggressive papillary middle ear tumor (APMET) and ELST. APMET was proposed as a type of temporal bone tumor. However, some authors have recognized APMET and ELST as synonymous $(4,13,24,25)$ and there seems to be confusion for these temporal tumors. Wenig and Heffner disagreed with APMET as distinct entity based on the facts of APMET showing similar clinical, radiologic and pathologic findings as ELST (2).

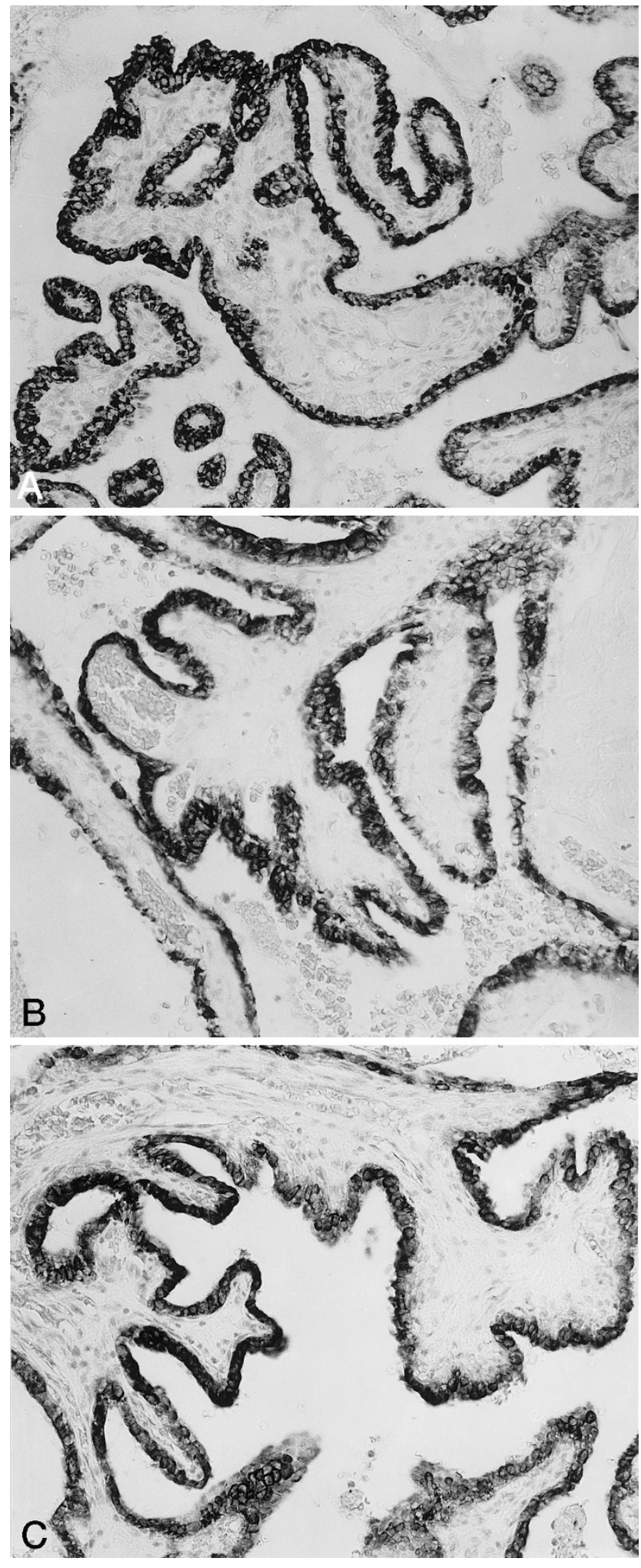

FIGURE 5. Immunohistochemistry for CK7 (A), CK8 (B), and CK19 (C) in endolymphatic sac tumor.

They concluded that tumors considered as APMET are ELST in fact and warned that ELST with endolymphatic sac epithelium origin is a distinct clinicopathologic entity and should not be confused with middle ear origin tumors and other temporal 


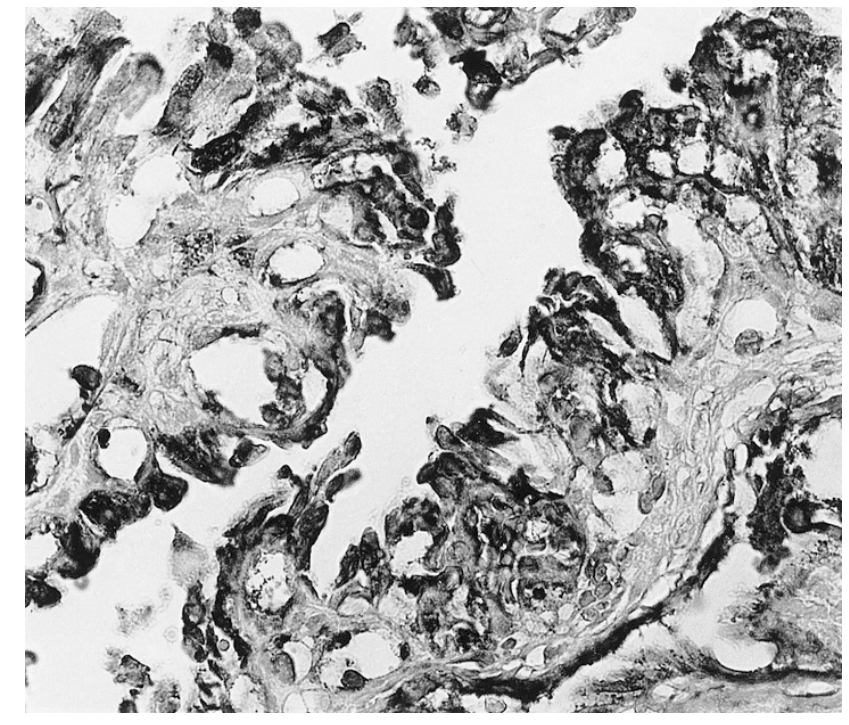

FIGURE 6. Vascular endothelial growth factor expression in tumor cells of endolymphatic sac tumor.

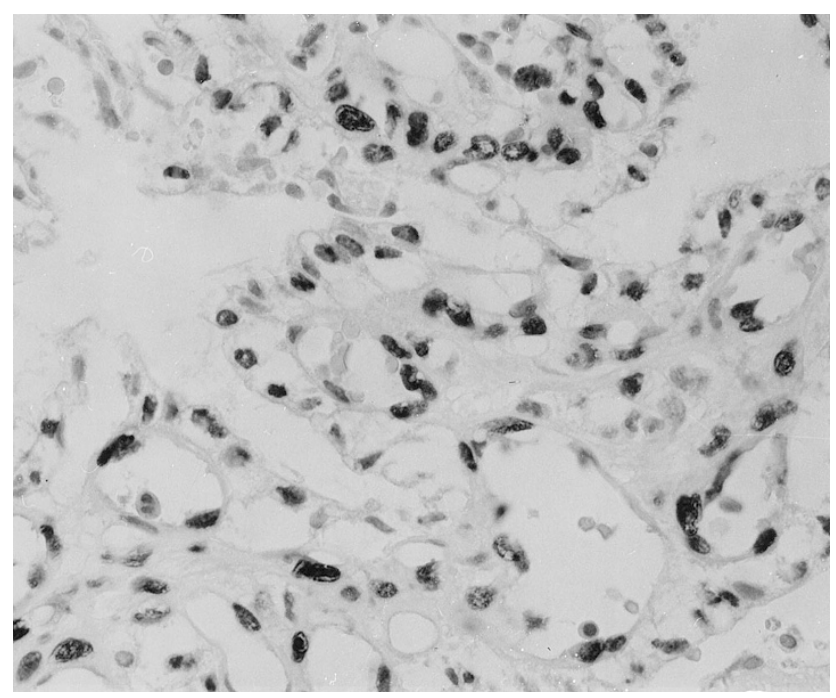

FIGURE 7. Focal high-density area for Ki-67 antigen in endolymphatic sac tumor.

tumors. Therefore, the term of APTTE may be carefully used. The present case showing posteriormedial petrous origin (1), bone destruction and papillary architecture corresponds to the distinct feature of ELST.

Expression of various CKs has been reported in many cancers, and some tumors have characteristic combinations (26-28). Many investigators documented that ELSTs commonly express CKs $(2-6,8)$, but most authors used single antibodies. Therefore, the expression pattern for various types of CKs has not been well researched. Paulus et al. (14) found expressions for CK8 and CK18 but not CK5, CK6, CK7 or CK19 in middle ear adenocarcinoma with neuroendocrine differentiation. This pattern is different from ours. Further cases must be studied before we will know whether the pattern of CK expression is useful to distinguish ELST from other temporal epithelial tumors, such as middle ear adenoma, papilloma of the middle ear, choroid plexus papilloma and metastatic carcinoma.

Recently, ELST has been identified as a manifestation of VHL. The present case with a cerebellar hemangioblastoma and family history of the same tumors is clinically identified as a VHL patient. Gaffey et al. (16) found a statistically significant occurrence of APMET (probably ELST) in VHL patients. Kempermann et al. (7) reviewed previous literature and indicated that ELST can grow bilaterally in VHL patients. Moreover, Tibbs et al. (24) proved mutation of VHL in ELST using singlestrand conformation polymorphism. These findings support the diagnosis of a temporal papillary tumor as ELST for a case with VHL such as ours.

VEGF is the most common promoter of angiogenesis; it induces endothelial proliferation and permeability. Various kinds of tumors associated with VHL are known to show VEGF expression. Brieger et al. (29) found immunoreactivity for VEGF in 22 of 29 renal cell carcinomas. Krieg et al. (30) recognized the presence of VEGF protein in all 11 hemangioblastomas of the central nervous system by Northern blot analysis. In papillary cystadenoma of the epididymis, the expression of VEGF mRNA was proved using in situ hybridization (31). These findings indicate that many tumors associated with VHL promote the production of VEGF protein. The present study revealed a strong VEGF expression in tumor cells of ELST, which suggests that VEGF overexpression has an important role for angiogenesis in ELST, much like that in other VHLassociated tumors.

ELSTs frequently show bone destruction and invasion. Heffner (1) previously reported that this tumor should be called a low-grade adenocarcinoma, but now he considered that this tumor is not malignant in the sense that it is locally infiltrative without metastasizing (2). However, reports on the proliferation potential of ELST are very few. Asano et al. (8) reported the LI of Ki-67 antigen in a longterm survival (more than 50 years) case of ELST. In their case, the LI in the area with papillary structure was less than $1 \%$, and the strongly pleomorphic area showed $3 \%$ positivity. On the other hand, in the present case, where the patient experienced the progression of ELST in 3 years, the LI of Ki-67 antigen was very high (48.9\%) in a focal area despite the lack of mitosis. This suggests that ELST may include a highly aggressive component although it shows generally a low proliferative activity. Wenig and Heffner (2) reported that a radical resection is the best treatment for this tumor. Reseachers seem not to agree on whether irradiation and chemother- 
apy are necessary in the case of ELST showing a high level of proliferation (2).

In conclusion, we found a strong VEGF expression in a case of ELST with VHL. This suggests that ELSTs have a similar angiogenetic mechanism as other VHL-associated tumors. A focal, highly proliferative lesion in the present tumor indicates that this case of ELST may contain a highly aggressive component. The ELST expressed various kinds of CKs, but further study is necessary to see which combination of CKs is characteristic for this tumor.

\section{REFERENCES}

1. Heffner DK. Low-grade adenocarcinoma of probable endolymphatic sac origin. A clinicopathologic study of 20 cases. Cancer 1989;64:2292-302.

2. Wenig BM, Heffner DK. Endolymphatic sac tumors: fact or fiction? Adv Anat Pathol 1996;3:378-87.

3. Gaffey MJ, Mills SE, Fechner RE, Intemann SR, Wick MR. Aggressive papillary middle-ear tumor. A clinicopathologic entity distinct from middle-ear adenoma. Am J Surg Pathol 1988;12:790-7.

4. Lavoie M, Morency RM. Low-grade papillary adenomatous tumors of the temporal bone: report of two cases and review of the literature. Mod Pathol 1995;8:603-8.

5. Levin RJ, Feghali JG, Morganstern N, Llena J, Bradley MK. Aggressive papillary tumors of the temporal bone: an immunohistochemical analysis in tissue culture. Laryngoscope 1996;106:144-7.

6. Kempermann G, Neumann HP, Scheremet R, Volk B, Mann W, Gilsbach J, et al. Deafness due to bilateral endolymphatic sac tumours in a case of von Hippel-Lindau syndrome. J Neurol Neurosurg Psychiatry 1996;61:318-20.

7. Kempermann G, Neumann HP, Volk B. Endolymphatic sac tumours. Histopathology 1998;33:2-10.

8. Asano K, Sekiya T, Hatayama T, Tanaka M, Takemura A, Suzuki S, et al. A case of endolymphatic sac tumor with long-term survival. Brain Tumor Pathol 1999;16:69-76.

9. Eby TL, Makek MS, Fisch U. Adenomas of the temporal bone. Ann Otol Rhinol Laryngol 1998;97:605-12.

10. Wassef M, Kanavaros P, Polivka M, Nemeth J, Monteil JP, Frachet B, et al. Middle ear adenoma. A tumor displaying mucinous and neuroendocrine differentiation. Am J Surg Pathol 1989;13:838-47.

11. Robson AK, Eveson JW, Smith IM, Maw AR. Papillary adenocarcinoma of the middle ear. J Layngol Otol 1990;104:91516.

12. Li JC, Brackmann DE, Lo WWM, Carverry JN, House JW. Reclassification of aggressive adenomatous mastoid neoplasms as endolymphatic sac tumors. Laryngoscope 1993; 103:1342-8.

13. Reijneveld J, Hanlo P, Groenewoud G, Jansen G, van Overbeeke K, Tulleken C. Endolymphatic sac tumor: a case report and review of the literature. Surg Neurol 1997;48:368-73.

14. Paulus W, Romstöck J, Weidenbecher M, Huk WJ, Fahlbusch R. Middle ear adenocarcinoma with intracranial extension. Case report. J Neurosurg 1999;90:555-8.

15. Poe DS, Tarlov EC, Thomas CB, Kveton JF. Aggressive papillary tumors of temporal bone. Otolaryngol Head Neck Surg $1993 ; 108: 80-6$.
16. Gaffey MJ, Mills SE, Boyd JC. Aggressive papillary tumor of middle ear/temporal bone and adnexal papillary cystadenoma. Manifestations of von Hippel-Lindau disease. Am J Surg Pathol 1994;18:1254-60.

17. Manski TJ, Heffner DK, Glenn GM, Patronas NJ, Pikus AT, Katz D, et al. A source of morbid hearing loss in von HippelLindau disease. JAMA 1997;277:1461-6.

18. Latif F, Tory K, Gnarra J, Yao M, Duh FM, Orcutt ML, et al. Identification of the von Hippel-Lindau disease tumor suppressor gene. Science 1993;260:1317-20.

19. Gnarra JR, Zhou S, Merrill MJ, Wagner JR, Krumm A, Papavassiliou E, et al. Post-transcriptional regulation of vascular endothelial growth factor mRNA by the product of the VHL tumor suppressor gene. Proc Natl Acad Sci USA 1996;93: 10589-94.

20. Wizigmann-Voos S, Breier G, Risau W, Plate KH. Upregulation of vascular endothelial growth factor and its receptors in von Hippel-Lindau disease-associated and sporadic hemangioblastomas. Cancer Res 1995;55:1358-64.

21. Roche PH, Dufour H, Figarella-Branger D, Pellet W. Endolymphatic sac tumors: report of three cases. Neurosurgery 1998;42:927-32.

22. Michaels L. The ear. In: Sternberg SS, editor. Histology for pathologist. 2nd ed. New York: Lippincott-Raven; 1997. p. 337-66.

23. Mills SE, Gaffey MJ, Frierson HF Jr. Atlas of tumor pathology. Tumors of the upper aerodigestive tract and ear. 3rd Series. Fascicle 26. Washington, DC: Armed Forces Institute of Pathology; 2000.

24. Tibbs RE Jr, Bowles AP Jr, Raila FA, Fratkin JD, Hutchins JB. Should endolymphatic sac tumors be considered part of the von Hippel-Lindau complex? Pathology case report. Neurosurgery 1997;40:848-55.

25. Polinsky MN, Brunberg JA, McKeever PE, Sandler HM, Telian S, Ross D. Aggressive papillary middle ear tumors: a report of two cases with review of the literature. Neurosurgery 1994; 35:493-7.

26. Hammar SP. Metastatic adenocarcinoma of unknown primary origin. Hum Pathol 1998;29:1393-402.

27. Fonseca E, Nesland JM, Höie J, Sobrinho-Simões M. Pattern of expression of intermediate cytokeratin filaments in the thyroid gland: an immunohistochemical study of simple and stratified epithelial-type cytokeratins. Virch Arch 1997;430: $239-45$.

28. Hirokawa M, Carney JA, Ohtsuki Y. Hyalinizing trabecular adenoma and papillary carcinoma of the thyroid gland express different cytokeratin patterns. Am J Surg Pathol 2000; 24:877-81.

29. Brieger J, Weidt EJ, Schirmacher P, Störkel S, Huber C, Decker HJ. Inverse regulation of vascular endothelial growth factor and VHL tumor suppressor gene in sporadic renal cell carcinomas is correlated with vascular growth: an in vivo study on 29 tumors. J Mol Med 1999;77:505-10.

30. Krieg M, Marti HH, Plate KH. Coexpression of erythropoietin and vascular endothelial growth factor in nervous system tumors associated with von Hippel-Lindau tumor suppressor gene loss of function. Blood 1998;92:3388-93.

31. Leung SY, Chan ASY, Wong MP, Yuen ST, Fan YW, Chung LP. Expression of vascular endothelial growth factor in von Hippel-Lindau syndrome-associated papillary cystadenoma of the epididymis. Hum Pathol 1998;29:1322-4. 